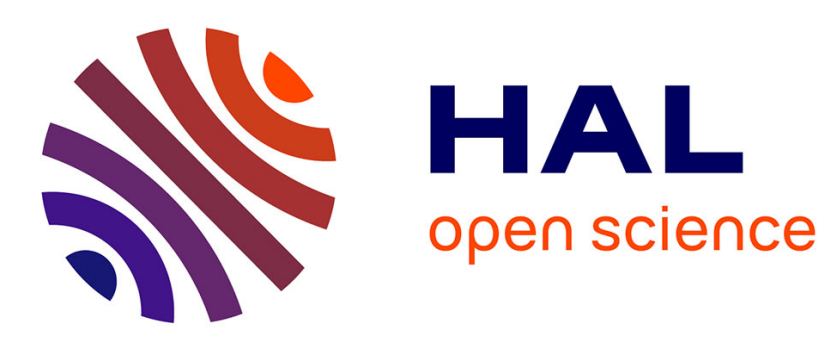

\title{
Tuning exchange-bias properties by thermal effects in a hard/soft bilayer
}

Thomas Hauet, S Mangin, F Montaigne, J. A. Borchers, Y Henry

\section{To cite this version:}

Thomas Hauet, S Mangin, F Montaigne, J. A. Borchers, Y Henry. Tuning exchange-bias properties by thermal effects in a hard/soft bilayer. Applied Physics Letters, 2007, 10.1063/1.2753108 . hal01345235

\section{HAL Id: hal-01345235 \\ https://hal.science/hal-01345235}

Submitted on 13 Jul 2016

HAL is a multi-disciplinary open access archive for the deposit and dissemination of scientific research documents, whether they are published or not. The documents may come from teaching and research institutions in France or abroad, or from public or private research centers.
L'archive ouverte pluridisciplinaire $\mathbf{H A L}$, est destinée au dépôt et à la diffusion de documents scientifiques de niveau recherche, publiés ou non, émanant des établissements d'enseignement et de recherche français ou étrangers, des laboratoires publics ou privés. 


\title{
Tuning exchange-bias properties by thermal effects in a hard/soft bilayer
}

\author{
T. Hauet, ${ }^{a}$ S. Mangin, and F. Montaigne \\ LPM, Nancy-University, CNRS, BP 239, F-54506 Vandoeuvre Cedex, France \\ J. A. Borchers \\ NIST Center for Neutron Research, Bldg. 235, 100 Bureau Drive, Stop 6102 Gaithersburg, \\ Maryland 20899-6102 \\ Y. Henry \\ IPCMS, CNRS, 23 Rue du Loess, BP 43, F-67034 Strasbourg Cedex 2, France
}

(Received 3 April 2007; accepted 7 June 2007; published online 12 July 2007)

\begin{abstract}
The effect of temperature on exchange-bias properties of an antiferromagnetically coupled hard/soft bilayer $\left(\mathrm{Tb}_{12} \mathrm{Fe}_{88} / \mathrm{Gd}_{40} \mathrm{Fe}_{60}\right)$ is studied. In a similar manner to its cooling field dependence, a continuous transition from a negative to a positive exchange-bias field is observed with increasing temperature. The changes of magnetic configuration responsible for this effect are studied, combining both magnetization and polarized neutron reflectivity measurements. The temperature is found to enhance the exchange-bias training effect as a result of the relaxation of an interface domain wall. The present study demonstrates that both temperature and cooling field may be used to tune the exchange field. (C) 2007 American Institute of Physics. [DOI: 10.1063/1.2753108]
\end{abstract}

For the last 15 years, the exchange-bias effect in antiferromagnetic/ferromagnetic (AF/FM) bilayers have been widely studied due to its technological applications and the specific physical mechanisms involved. ${ }^{1}$ When an exchange-biased system is cooled below a certain temperature known as the blocking temperature $\left(T_{B}\right)$, the hysteresis loop of the FM layer is shifted toward a field $H_{E}$, named the exchange-bias field. Bilayers showing an antiferromagnetic interfacial exchange coupling are particularly interesting since $H_{E}$ shows a continuous transition from negative to positive with increasing cooling field $\left(H_{\mathrm{cf}}\right)^{2,3}$ Recently, a similar transition has also been observed as a function of temperature in an $\mathrm{AF} / \mathrm{FM}$ system $\mathrm{FeZnF}_{2} / \mathrm{Co}$ (Ref. 4) and in an $\mathrm{AF} /$ ferrimagnetic bilayer $\mathrm{NiCoO} / \mathrm{GdFe}^{5}$ In both cases, the given explanation involves a reorientation of the magnetic configuration inside the $\mathrm{AF}$ layer as the temperature changes. However, the type of reorientation mechanism proposed is quite different in the two cases. For the first one, it was assumed that an unstable domain structure is formed in the AF, which then relaxes. For $\mathrm{NiCoO} / \mathrm{GdFe}$, a memory effect is proposed, ${ }^{6}$ which takes into account the evolution of an in-plane domain wall localized in the AF. The main difficulty in validating either one of these theories is that the interface magnetic configurations in AF layers are very difficult to probe mainly because the antiferromagnetic layer has almost no net magnetization. To go beyond this problem, we have studied a ferrimagnetic/ferrimagnetic, hard/soft bilayer, namely, $\mathrm{TbFe} / \mathrm{GdFe}$, which mimics antiferromagnetically coupled AF/FM systems. Such a system is a model as its magnetic configuration is easy to probe with conventional means. Recently, we were able to prove that during field cooling, a partial interfacial domain wall is frozen in the $\mathrm{TbFe}$ layer. The resulting exchange-bias field is then determined only by the orientation of the quenched TbFe magnetization at the interface, which rotates continuously from antiparallel to the cooling field direction to parallel as $H_{\mathrm{cf}}$ increases. ${ }^{7,8}$ We have used this system to study the exchange-

\footnotetext{
a)Electronic mail: hauet@1pm.u-nancy.fr
}

bias training effect. This effect is characterized by the evolution of the hysteresis loop with the number of field cycles. ${ }^{9,10}$ Using polarized neutron reflectivity (PNR), we demonstrated that these changes in $H_{E}$ are caused by irreversible modifications of the magnetic configuration in TbFe. ${ }^{11}$

In this letter, we first illustrate how the exchange-bias phenomena in $\mathrm{TbFe} / \mathrm{GdFe}$ depend not only on the cooling field or on the number of field cycles performed, but also on temperature. We show that the variations of $H_{E}$ as a function of the temperature are comparable to those observed in the systems mentioned above. We then use both conventional magnetization measurements and PNR to probe the changes in the magnetic configuration. We finally demonstrate that the temperature variations in the exchange-bias field are explained by thermally activated relaxation of an interfacial domain wall (iDW).

The sample used for the described experiments consists of glass $/ \mathrm{Gd}_{40} \mathrm{Fe}_{60} \quad(100 \mathrm{~nm}) / \mathrm{Tb}_{12} \mathrm{Fe}_{88} \quad(50 \mathrm{~nm}) / \mathrm{Al}$ $(4.5 \mathrm{~nm}) / \mathrm{Al}_{2} \mathrm{O}_{3}(3.5 \mathrm{~nm})$. Due to the growth conditions, ${ }^{7,8}$ $\mathrm{Gd}_{40} \mathrm{Fe}_{60}$ and $\mathrm{Tb}_{12} \mathrm{Fe}_{88}$ are amorphous and antiferromagnetically exchange coupled across their common interface. After cooling this sample in a field $H_{\text {cf }}$ from room temperature down to different temperatures between 5 and $300 \mathrm{~K}$, three successive hysteresis loops, between +20 and $-20 \mathrm{mT}$, were recorded with a commercial SQUID magnetometer. Figure 1(a) shows the evolution of the exchange-bias field of the first and the third loops as a function of the temperature $(T)$ for a cooling field of $H_{\mathrm{cf}}=100 \mathrm{mT}$. We note that after three cycles no more changes were observed, regardless of the temperature and cooling field. For both the first and the third loops, the exchange-bias field shows a transition from negative to positive as the temperature increases. However, the difference between $H_{E}^{1}$ and $H_{E}^{3}$, i.e., the evolution of the exchange-bias properties with the number of cycles, reveals training effect processes. It appears that this difference between $H_{E}^{1}$ and $H_{E}^{3}$ increases with increasing $T$ until $H_{E}^{3}$ reaches the maximum value $+H_{E}^{\max }$. Figure 1(b) compares the variations of $H_{E}^{3}$ with $T$ for various $H_{\text {cf. }}$. Three particular be- 


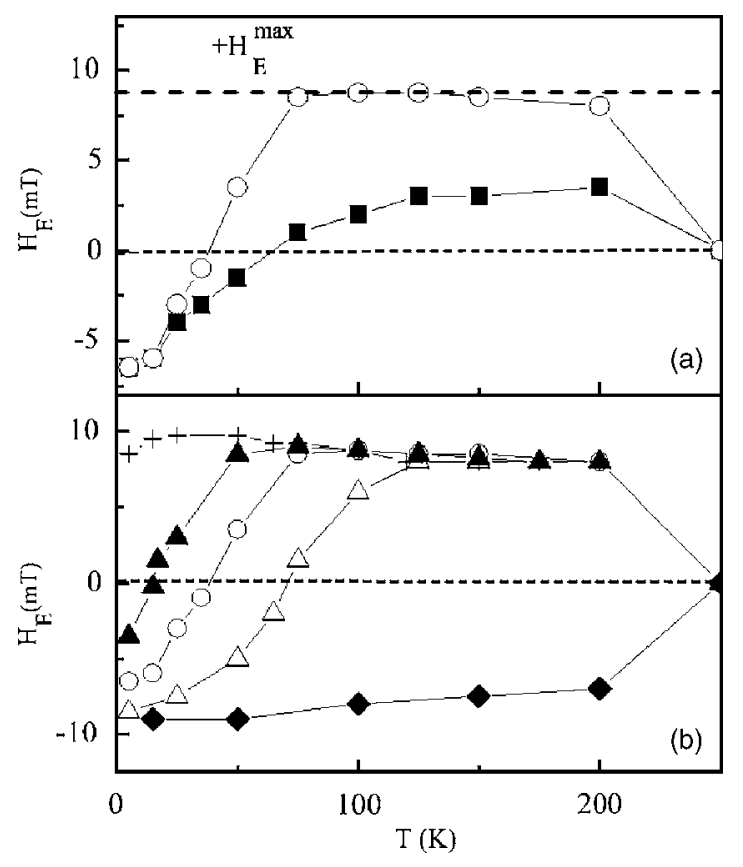

FIG. 1. (a) Temperature dependence of the exchange-bias fields of the first hysteresis loop $H_{E}^{1}$ (full squares) and third hysteresis loop $H_{E}^{3}$ (open circles) after cooling the sample in $100 \mathrm{mT}$. (b) Exchange-bias field of the third hysteresis loop $H_{E}^{3}$ as a function of temperature after cooling in $H_{\mathrm{cf}}=+3 \mathrm{mT}$ (full diamonds), $+50 \mathrm{mT}$ (open triangles), $+100 \mathrm{mT}$ (open circles), $+200 \mathrm{mT}$ (full triangles), and $+1.0 \mathrm{~T}$ (crosses).

haviors are observed. For a very low cooling field, e.g., $3 \mathrm{mT}, H_{E}^{1}=H_{E}^{3}=-H_{E}^{\max }$ for $T<T_{B}$ near $250 \mathrm{~K}$. For moderate cooling fields, e.g., 50, 100, or $200 \mathrm{mT}$, an evolution of the exchange-bias value appears from the first loop to the third one and a continuous transition of $H_{E}^{3}$ from negative to positive is observed as the temperature increases. In the case of larger cooling fields, e.g., 1.0 T, $H_{E}^{3}=H_{E}^{1}=+H_{E}^{\max }$ before decreasing to $0 \mathrm{mT}$ at the blocking temperature.

PNR is ideally suited to elucidate the origin of these peculiar behaviors as it provides a depth profile of both the sample structure and the vector magnetization. ${ }^{12,13}$ Our experiments were performed at the NG-1 reflectometer at the NIST Center for Neutron Research. ${ }^{14}$ For these experiments, the neutron polarization direction and the applied field were maintained parallel, in the plane of the sample. A supermirror polarizer and analyzer selected one of the neutron spin states of the incident and scattered neutrons, and a pair of spin flippers orient the neutrons parallel $(+)$ or antiparallel $(-)$ to the field. The four PNR coefficients $R^{++}, R^{--}, R^{+-}$, and $R^{-+}$ were thus measured as a function of neutron wave vector $Q$. The data were corrected for the polarization efficiencies, which exceeded $96 \%$, as well as for the instrumental background. The non-spin-flip cross sections $\left(R^{++}\right.$and $\left.R^{-}\right)$are sensitive to the chemical structure and the moment projection parallel to the field, whereas the spin-flip cross sections $\left(R^{+-}\right.$and $\left.R^{-+}\right)$are sensitive to the moment projection perpendicular to the field. Then, depth-dependent magnetic and structural profiles can be deduced by fitting PNR data with a model for the scattering length density. In the present case, we used REFLPAK. ${ }^{15}$ Due to the large Gd absorption, the fits are particularly sensitive to the magnetic configuration in the bulk of the $\mathrm{TbFe}$ layer and at the $\mathrm{TbFe} / \mathrm{GdFe}$ interface. Keeping structural parameters constant and considering a temperature variation of the $\mathrm{TbFe}$ and $\mathrm{GdFe}$ magnetization consistent with the bulk, experimental data were remarkably

Downloaded 13 Aug 2007 to 69.26 .32 .67 . Redistribution subject to

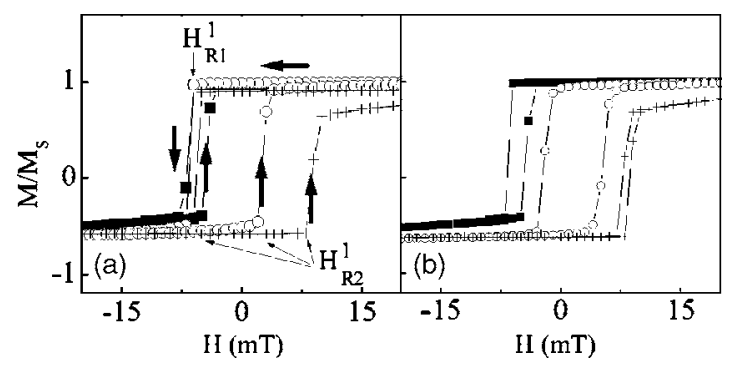

FIG. 2. (a) First and (b) third hysteresis loops measured after cooling the $\mathrm{GdFe} / \mathrm{TbFe}$ bilayer in $H_{\mathrm{cf}}=+100 \mathrm{mT}$ from room temperature down to $15 \mathrm{~K}$ (full squares), $50 \mathrm{~K}$ (open circles), and $100 \mathrm{~K}$ (crosses).

well fitted with $\chi^{2}$ values less than 10 . For our PNR investigations, we focus on the $100 \mathrm{mT}$ cooling field case.

Figures 2(a) and 2(b) show, respectively, the first and third magnetization loops recorded at 15,50 , and $100 \mathrm{~K}$, after cooling in $100 \mathrm{mT}$. The first GdFe magnetization reversal at $H_{R 1}^{1}=-8.5 \mathrm{mT}$ is seen to be nearly independent of $T$. In contrast, the second $\mathrm{GdFe}$ magnetization reversal at $H_{R 2}^{1}$, along the ascending branch of the cycle depends on temperature. These differences lead to the evolution of $H_{E}^{1}$ with temperature shown in Fig. 1(a) and, finally, to very different $H_{E}^{3}$ values for each temperature [Fig. 2(b)]. PNR measurements were first carried out at 15, 50, and $100 \mathrm{~K}$ [Fig. 3(a)] in a field of $+20 \mathrm{mT}$, in order to determine the initial magnetic configuration of the bilayer immediately after cooling. As illustrated in Fig. 3(b), it is found independent of temperature. It consists of an interface domain wall, mainly located in $\mathrm{TbFe}$, with the interfacial $\mathrm{TbFe}$ magnetization oriented at
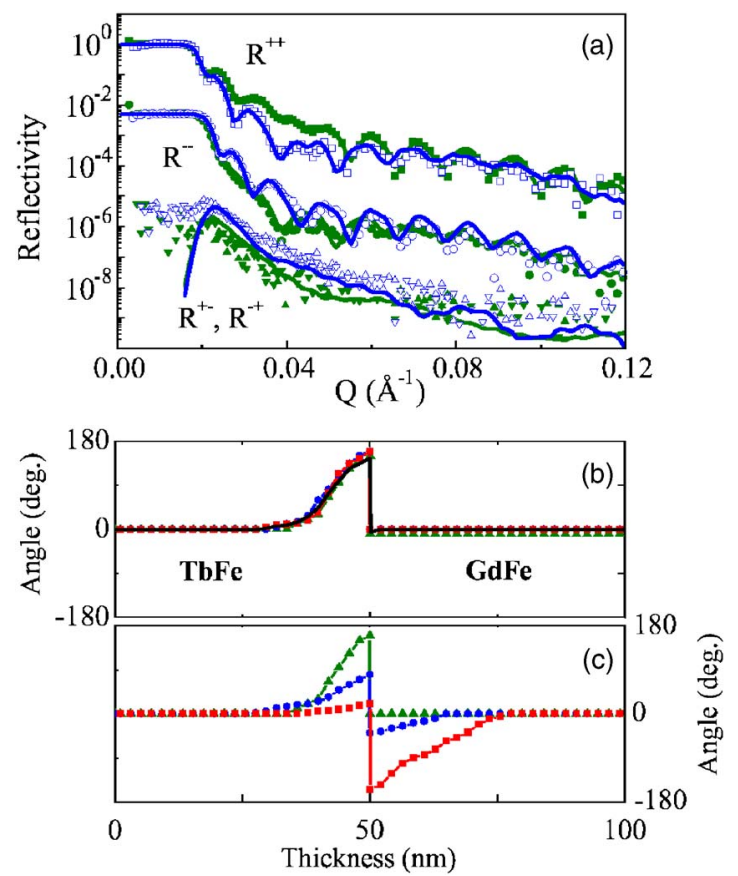

FIG. 3. (a) PNR curves recorded in $H=+20 \mathrm{mT}$ after cooling the sample from room temperature down to $100 \mathrm{~K}$ in $+100 \mathrm{mT}$ : (full green symbols) before the first GdFe reversal and (open blue symbols) after cycling the applied field three times between +20 and $-20 \mathrm{mT}$. $R^{--}$and $R^{+-}$(as $R^{-+}$) are offset from $R^{++}$by one and two orders of magnitude, respectively. The lines are the corresponding fits. [(b) and (c)] Depth dependence of the magnetization angle in $H=+20 \mathrm{mT}$ at $15 \mathrm{~K}$ (green triangles), $50 \mathrm{~K}$ (blue circles), and $100 \mathrm{~K}$ (red squares): (b) immediately after cooling in $100 \mathrm{mT}$ and (c) after three $\pm 20 \mathrm{mT}$ field cycles. The black line in (b) is the magnetic con-

figuration prior to cooling $(T=300 \mathrm{~K}, H=+100 \mathrm{mT})$. 
an angle of $\theta_{\mathrm{TbFe}}^{i}=150^{\circ}$ from the positive field direction. $\mathrm{GdFe}$ for its part is magnetized uniformly along the positive field direction. This configuration is identical to that formed when applying $100 \mathrm{mT}$, prior to cooling [Fig. 3(b)], which confirms that the magnetization distribution of $\mathrm{TbFe}$ "freezes" as the temperature is reduced. ${ }^{7} H_{\mathrm{R} 1}^{1}$ is essentially determined by the orientation of the $\mathrm{TbFe}$ magnetization at the interface. ${ }^{8}$ This orientation $\left(\theta_{\mathrm{TbFe}}^{i}\right)$ is independent of temperature, so is $H_{\mathrm{R} 1}^{1}$. To understand why, unlike $H_{\mathrm{R} 1}^{1}$, the second GdFe reversal field $H_{\mathrm{R} 2}^{1}$ [Fig. 2(a)] and, subsequently, $H_{E}^{3}$ (Fig. 1) strongly vary with temperature, PNR measurements were also performed after completion of the three $\pm 20 \mathrm{mT}$ field cycles. Figure 3(a) compares the reflectivity coefficients $\left(R^{++}, R^{--}, R^{+-}\right.$, and $\left.R^{-+}\right)$at $100 \mathrm{~K}$ before and after cycling the applied field. Strong differences are clearly visible between the two sets of data, which reveals that the magnetic configuration in the bilayer has evolved. After cycling, $\mathrm{TbFe}$ is now magnetized in a quasiuniform manner along the positive field direction and an iDW is forced into the GdFe layer to satisfy the interface antiferromagnetic exchange coupling [Fig. 3(c)]. The TbFe magnetization originally oriented at $\theta_{\mathrm{TbFe}}^{i}=150^{\circ}$ now points at $\theta_{\mathrm{TbFe}}^{i}=20^{\circ}$, which is consistent with $H_{E}^{3}=+8 \mathrm{mT}$ being close to $+H_{E}^{\max } .{ }^{8}$ It is clear in Fig. 3(c) that the new configuration formed after field cycling depends on temperature, especially near the interface, hence the variation of $H_{E}^{3}$ with $T$. For the intermediate temperature of $50 \mathrm{~K}$, the iDW is located in both the GdFe and $\mathrm{TbFe}$ layers. The interface $\mathrm{TbFe}$ magnetization is almost perpendicular to the field direction $\left(\theta_{\mathrm{TbFe}}^{i}=80^{\circ}\right)$, which results in the small exchange-bias field of $H_{E}^{3}=+1.5 \mathrm{mT}$. Finally, at the lowest temperature of $15 \mathrm{~K}$, no change in the $\mathrm{TbFe}$ magnetization is seen, which agrees with $H_{E}^{3}=H_{E}^{1}$ [Fig. 1].

Our present results confirm that successive hysteresis loops induce an irreversible evolution of a metastable iDW that is created at the $\mathrm{GdFe} / \mathrm{TbFe}$ interface during the field cooling. This relaxation always tends to displace the iDW from the hard layer to the soft layer in order to decrease the total bilayer energy. Moreover, our results prove that the extent of the iDW displacement increases as $T$ grows. The training effect thus has a thermal activation, and this effect is responsible for the transition of $H_{E}^{3}$ from negative to positive as the temperature increases for $H_{\mathrm{cf}}=50,100$, and $200 \mathrm{mT}$ [Fig. 1(b)]. The continuous nature of these transitions is remarkable and comes from the progressive translation of the part of the iDW originally located in $\mathrm{TbFe}$ into the GdFe layer. Moreover, the dependence of $H_{E}^{3}(T)$ on cooling field [Fig. 1(b)] comes from the fact that, as $H_{\mathrm{cf}}$ increases, the frozen iDW is increasingly more compressed ${ }^{11}$ and its stored energy increases. Since the stability of the iDW has decreased, less thermal energy is required to reach the most stable state corresponding to $H_{E}=+H_{E}^{\max }$. Finally, the same reasoning explains the two extreme regimes observed in Fig. 1(b). In the first case of $H_{\mathrm{cf}}=3 \mathrm{mT}$, the energy stored in the $\mathrm{TbFe}$ layer is minimum because its frozen magnetization is uniformly antiparallel to the GdFe magnetization. Therefore, no training occurs and the exchange-bias field is constant $\left(H_{E}^{1}=H_{E}^{3}=-H_{E}^{\max }\right)$ for all temperatures below $T_{B}$. In the second case of $H_{\mathrm{cf}}=1.0 \mathrm{~T}$, the frozen iDW is so thin, i.e., $\theta_{\mathrm{TbFe}}^{i}=0^{\circ}$, that the training effect leads to the most stable state regardless of the temperature. Thus, $H_{E}^{3}=H_{E}^{1}=+H_{E}^{\max }$ at all temperatures.

In conclusion, we have studied the evolution of the exchange-bias field of a hard/soft, TbFe/GdFd bilayer that mimics antiferromagnetically coupled AF/FM systems. Over a large range of cooling field, $H_{E}$ was found to vary continuously from negative to positive as the temperature increases. Polarized neutron reflectivity, combined with magnetization measurements, proved that this feature is due to a temperature dependent training phenomenon. This effect originates from the relaxation, i.e., progressive untwisting, of a frozen interfacial domain wall located in the TbFe layer. The energy of the iDW is highly sensitive to the interplay between cooling field $H_{\mathrm{cf}}$ and temperature, and the relaxation of the iDW can be continuously controlled as a result. Our results thus reveal an efficient way to tune exchange-bias properties with both $H_{\mathrm{cf}}$ and temperature.

The authors thank W.-C. Chen and D. Pierre for help with the experiments and C. F. Majkrzak for helpful discussions.

${ }^{1}$ J. Nogues and I. K. Schuller, J. Magn. Magn. Mater. 192203 (1999).

${ }^{2}$ J. Nogues, D. Lederman, T. J. Moran, and I. K. Schuller, Phys. Rev. Lett. 76, 4624 (1996).

${ }^{3}$ J. Nogues, C. Leighton and I. K. Schuller, Phys. Rev. B 61, 1315 (2000). ${ }^{4}$ H. Shi, D. Lederman, N. R. Dilley, R. C. Black, J. Diedrichs, K. Jensen, and M. B. Simmonds, J. Appl. Phys. 93, 8600 (2003).

${ }^{5}$ D. Z. Yang, J. Du, L. Sun, X. S. Wu, X. X. Zhang, and S. M. Zhou, Phys. Rev. B 71, 144417 (2005).

${ }^{6}$ N. J. Gokemeijer, J. W. Cai, and C. L. Chien, Phys. Rev. B 60, 3033 (1999).

${ }^{7}$ S. Mangin, F. Montaigne, and A. Schuhl, Phys. Rev. B 68, 140404 (2003).

${ }^{8}$ Y. Henry, S. Mangin, T. Hauet, and F. Montaigne, Phys. Rev. B 73, 134420 (2006).

${ }^{9}$ A. Hochstrat, Ch. Bineck, and W. Kleemann, Phys. Rev. B 66, 092409 (2002).

${ }^{10}$ J. Keller, P. Miltényi, B. Beschoten, G. Güntherodt, U. Nowak, and K. D. Usadel, Phys. Rev. B 66, 014430 (2002).

${ }^{11}$ T. Hauet, J. A. Borchers, Ph. Mangin, Y. Henry, and S. Mangin, Phys. Rev. Lett. 96, 067207 (2006).

${ }^{12}$ M. R. Fitzsimmons, S. D. Bader, J. A. Borchers, G. P. Felcher, J. K. Furdyna, A. Hoffmann, J. B. Kortright, Ivan K. Schuller, T. C. Schulthess, S. K. Sinha, M. F. Toney, D. Weller, and S. Wolf, J. Magn. Magn. Mater. 271, 103 (2004)

${ }^{13}$ K. V. O'Donovan, J. A. Borchers, C. F. Majkrzak, O. Hellwig, and E. E. Fullerton, Phys. Rev. Lett. 88, 067201 (2002).

${ }^{14}$ C. F. Majkrzak, Physica B 221, 342 (1996).

${ }^{15} \mathrm{http} / / /$ www.ncnr.nist.gov/reflpak 